\title{
Féeries
}

Études sur le conte merveilleux, XVII $-\mathrm{XIX}{ }^{\mathrm{e}}$ siècle

\section{Les fées de Romagnesi et Procope-couteaux (1736) entre Perrault et Marivaux}

\section{Nathalie Rizzoni}

\section{(2) OpenEdition}

1 Journals

\section{Édition électronique}

URL : http://journals.openedition.org/feeries/343

DOI : $10.4000 /$ feeries.343

ISSN : 1957-7753

Éditeur

UGA Éditions/Université Grenoble Alpes

\section{Édition imprimée}

Date de publication : 1 octobre 2007

Pagination : 131-154

ISBN : 978-2-84310-101-4

ISSN : $1766-2842$

\section{Référence électronique}

Nathalie Rizzoni, «Les fées de Romagnesi et Procope-couteaux (1736) entre Perrault et Marivaux », Féeries [En ligne], 4 | 2007, mis en ligne le 04 novembre 2008, consulté le 08 septembre 2020. URL http://journals.openedition.org/feeries/343 ; DOI : https://doi.org/10.4000/feeries.343

Ce document a été généré automatiquement le 8 septembre 2020

(c) Féeries 


\title{
Les fées de Romagnesi et Procope- couteaux (1736) entre Perrault et Marivaux
}

\author{
Nathalie Rizzoni
}

NOUVEAU THE'ATRE ITALIEN.

L E S

F E E $S$,

Comedie en trois Aites.

Repréfentée pour la premiere fois par les Comediens Italiens ordinaires du Roi, le $1_{4}$ Juillet 1736.

Par Mefieurs ROMAGNESI Of C***

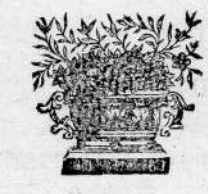

A P A R IS,

Chez BRIA s s o N, rue Saint Jacques: à la Science.

En ce samedi 14 juillet 1736, plus de cinq cents spectateurs ${ }^{1}$ se pressent à l'angle de la rue Mauconseil ${ }^{2}$ et de la rue Française à Paris pour découvrir, le jour de sa création, la nouvelle comédie de Jean-Antoine Romagnesi et Michel Procope-Couteaux. Est-ce le titre de la pièce, Les Fées, qui les attire en grand nombre à l'Hôtel de Bourgogne ce jourlà? ou bien est-ce le nom de l'un des auteurs, Romagnesi, dramaturge fécond qui 
compte déjà cinquante-cinq pièces à son actif sur cette scène et qui n'est pas moins apprécié en tant que comédien depuis ses débuts dans la troupe italienne, une dizaine d'années auparavant? L'une aussi bien que l'autre hypothèse expliquent sans doute ce que l'on peut considérer, au regard de la fréquentation moyenne pour une comédie de l'époque (environ trois cent vingt spectateurs) comme une belle affluence. La pièce nouvelle n'a d'ailleurs pas déçu l'attente de son public puisque le surlendemain, lorsqu'elle est rejouée, puis le 18 et le 21 juillet, l'affluence sera plus grande encore, avec respectivement 665,559 et 626 spectateurs.

2 Représentée à la Cour de Versailles à l'automne (le 20 octobre 1736), reprise chaque année pendant près de vingt ans (jusqu'au 19 mai 1754³) à la Comédie-Italienne, Les Fées auront suscité un intérêt soutenu et durable auprès du public, permanence de popularité peu commune pour une œuvre se réclamant de la veine féerique, dont les effets spectaculaires s'éventent habituellement sans tarder. Pour ne prendre que deux exemples significatifs, chez les Italiens toujours, ni la petite comédie en un acte Le Conte de fées (du même Romagnesi en société avec Lélio le fils) jouée un an plus tôt, ni le canevas italien Les Fées rivales (de Véronèse) donné une dizaine d'années plus tard ne connurent pareille fortune ${ }^{4}$ bien qu'elles eurent toutes deux un succès incontestable dans leur nouveauté 5 .

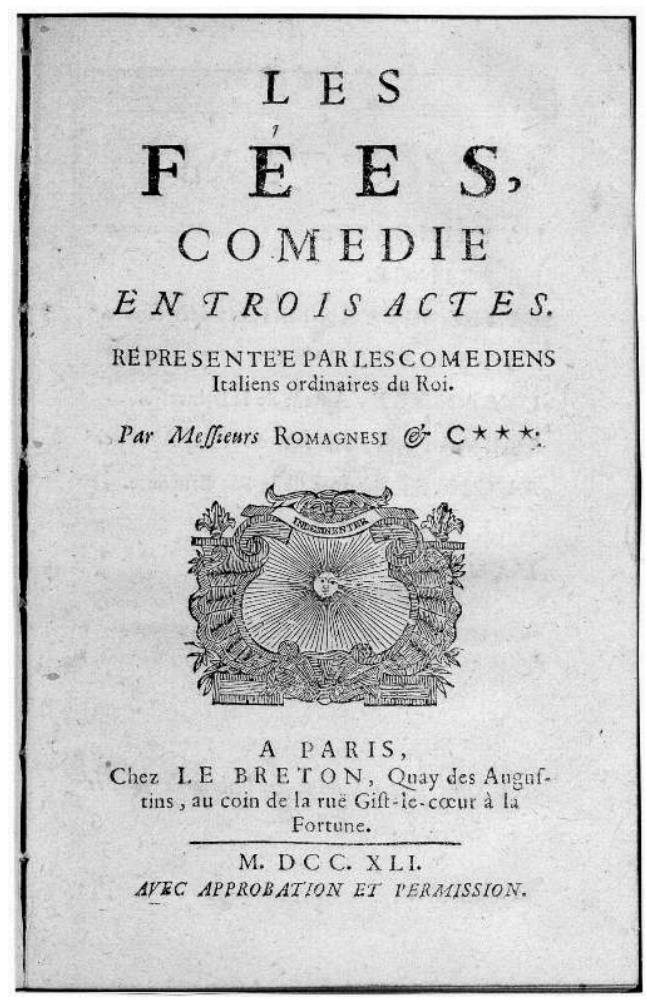

Il s'ajoute à cette différence que ni Le Conte de Fées ni Les Fées rivales n'ont eu l'honneur de l'édition (il était courant que ce genre de pièces ne soit pas publié), alors que la comédie des Fées connut au moins deux éditions isolées (en 1736 et en $1741^{6}$ ) et fut sélectionnée - signe de distinction - pour figurer dans le recueil en dix volumes du Nouveau Théâtre Italien... en $1753^{7}$ et encore en 1824 dans Fin du Répertoire du Théâtre français (t. 4, Comédies en prose). C'est dire que notre pièce a exercé sur le public une séduction singulière dont nous nous efforcerons de percer le secret ${ }^{8}$.

Lorsqu'ils intitulent leur comédie Les Fées, Romagnesi et Procope-Couteaux entendent à coup sûr amener au Théâtre-Italien cette part importante des spectateurs qui ne se 
lasse pas de retrouver sur les planches les génies, les magiciennes et les divinités imaginaires, merveilleusement campés par Perrault, $M^{\text {me }}$ d'Aulnoy, $M^{\text {me }} \mathrm{d}^{\text {'Auneuil, ou }}$ $\mathrm{M}^{\text {lle }}$ de La Force dans leurs recueils. Les théâtres de la Foire se sont fait une spécialité de la transposition des univers fantaisistes de ces conteurs depuis la fin du dix-septième siècle; et cette veine ne s'épuise pas au cours des années 1730 , comme en témoignent des pièces au titre aussi évocateur que L'Épreuve des fées $(1732)^{9}$, Le Palais enchanté $(1734)^{10}$, La Fée Marotte $(1734)^{11}$, L'T̂le des fées ou Le Géant aux marionnettes $(1735)^{12}$, La Fée Bienfaisante $(1736)^{13}$, Polichinelle roi des fées $(1737)^{14}$, La Fée Brochure $(1737)^{15}$, etc. Pour contrer la concurrence des Forains et satisfaire cette facette du goût du public, les dramaturges de l'Hôtel de Bourgogne, au premier rang desquels Romagnesi, se résignent à renouer avec l'inspiration débridée des productions de l'Ancien Théâtre Italien. À leur tour, ils font se côtoyer mondes fabuleux et monde réel dans leur répertoire et, après la pièce Le Conte de Fées déjà citée, la comédie Les Fées se présente bien comme un jalon marquant dans cette conquête toujours recommencée du public.

En découvrant Les Fées de 1736, les «théâtromanes » de l'époque ont pu se souvenir que deux comédies au moins avaient déjà été jouées sous ce titre à la fin du dix-septième siècle. L'une, de Dufresny et Brugière de Barante, fut la dernière pièce créée sur l'Ancien Théâtre Italien avant sa fermeture en 1697. L'autre, de Dancourt, était une commande royale, d'abord représentée au Château de Fontainebleau, puis donnée à la Comédie-Française en $1699^{16}$. Si tous les spectateurs ne sont pas en âge d'avoir assisté à l'une ou à l'autre de leurs représentations - ces pièces ne furent jamais reprises avant $1736^{17}$ - certains les auront peut-être lues dans les éditions successives du Théâtre Italien de Gherardi d'une part et des Euvres de Dancourt d'autre part: mais quel lien pouvaient-ils attendre entre la nouvelle pièce et ces deux comédies disparates que rien, sinon le titre, ne rapprochait?

6 Est-ce plutôt alors du côté de Perrault lui-même que les spectateurs étaient invités à chercher une piste? Notre comédie est-elle une adaptation dramatique de son conte intitulé Les Fées précisément, publié dans les Histoires ou contes du temps passé ? ${ }^{18}$ Malgré l'homonymie, ce n'est pas ce conte de Perrault qui en est la source (d'ailleurs ni la pièce de Dufresny et Brugière de Barante, ni celle de Dancourt ne s'y référaient), mais en réalité un autre conte du même auteur, Riquet à la houppe, l'histoire de ce prince « si laid et si mal fait, qu'on douta longtemps s'il avait une forme humaine ${ }^{19}$ ".

7 Rappelons le conte dans ses grandes lignes. Afin d'atténuer sa monstruosité physique, une Fée dote un jeune Prince d'un esprit brillant en même temps qu'elle lui accorde le pouvoir de rendre spirituelle la personne qu'il aimera le mieux. Quelques années plus tard, la même Fée condamne à la plus parfaite stupidité une jeune Princesse dont la beauté est extraordinaire, tandis qu'elle gratifie sa sœur cadette d'un esprit lumineux afin d'en faire oublier la laideur. En grandissant, la disgrâce de la cadette s'avère moins handicapante que la bêtise de l'aînée, qui met toutes les compagnies en fuite. « Un jour qu'elle s'était retirée dans un bois pour y plaindre son malheur ${ }^{20}$ ", la belle Princesse rencontre le Prince Riquet qui lui donne autant d'esprit qu'elle en souhaite contre la promesse de se faire épouser d'elle un an plus tard. Courtisée par les princes les plus puissants depuis qu'elle est aussi intelligente que belle, la Princesse oublie sa rencontre et sa promesse, jusqu'au jour où le hasard mène de nouveau ses pas dans le bois. Et de nouveau elle tombe sur le Prince Riquet, qui lui rappelle son engagement. Tandis qu'elle tente de le convaincre de lui rendre sa parole, Riquet lui révèle qu'elle a reçu de la Fée à sa naissance le pouvoir de rendre beau celui qu'elle aimera; ce qu'elle fait 
aussitôt avant de l'épouser. Et le conteur de conclure : «quelques-uns assurent que ce ne furent point les charmes de la Fée qui opérèrent, mais que l'amour seul fit cette métamorphose ${ }^{21}$ ».

On imagine sans peine comment Romagnesi pouvait s'emparer de cette fable, pour en faire une petite machine propre à soutenir la concurrence des forains. Ce dramaturge aguerri ne connaissait-il pas les ressorts de leur succès, lui qui avait débuté comme acteur au jeu de son oncle Octave (Jean-Baptiste Constantini) durant la Foire SaintGermain 1712, puis comme auteur l'année suivante, collaborant ensuite à son entreprise foraine jusqu'en 1716 ?

On entrevoit la petite pièce en un acte et en une quinzaine de scènes que Romagnesi aurait pu bâtir à partir du conte de Perrault, depuis le moment où les deux Princesses entrent en rivalité jusqu'au tableau final des noces, en passant par les monologues de la Princesse, ses duos avec le Prince Riquet, avec le roi son père, et bien sûr les scènes à tiroir, piquantes à souhait, de déclarations d'amour des prétendants couronnés.

Romagnesi avait également été à bonne école pour exploiter toutes les ressources de la mise en scène spectaculaire auxquelles le conte se prêtait naturellement. On songe aux changements de décors surprenants, avec ici un va-et-vient entre les salles du Palais du Roi et le bois où la Princesse fait la connaissance de Riquet, sans omettre la cuisine où l'on s'affaire pour la préparation des noces, au milieu de laquelle trône une « table fort longue » comme le précise Perrault. On songe encore à l'effet de machines prodigieuses, permettant le surgissement des entrailles de la terre de la fameuse grande cuisine à ciel ouvert. Des costumes somptueux pouvaient souligner la beauté de la Princesse, signifier la puissance des princes rivaux, tandis que la magnificence des habits de Riquet - par deux fois soulignée dans le conte - eût été à la mesure de sa bravoure. Une batterie d'accessoires également prélevés dans le conte pouvait renforcer le pittoresque de certaines scènes: les porcelaines que la princesse brise par maladresse, ses portraits qui courent partout dans le monde, les ustensiles de cuisine, les lardoires et les queues de renard, attributs indissociables de l'uniforme des cuisiniers. On songe enfin aux mouvements de foule susceptibles d'engendrer de véritables ballets en musique, que Perrault orchestre lui-même lorsqu'il décrit l'agitation des marmitons et de la bande des «vingt ou trente rôtisseurs" qui travaillent «en cadence au son d'une chanson harmonieuse ${ }^{22} »$.

11 Nos dramaturges ont renoncé, pourtant, à cette option dramaturgique qui les aurait conduits à privilégier le caractère enchanteur du conte qui les inspire. Dans leur comédie, le merveilleux est certes présent, mais ses manifestations matérielles sont des plus discrètes. Tout juste sait-on, grâce à une didascalie inaugurale, que «La scène se passe dans le Palais de la fée Bruyante » et l'on constate que le décor ne change jamais tout au long de la pièce. Pour ce qui est des accessoires, le portrait de la Princesse, trouvé un jour par le Prince à son réveil, est évoqué plusieurs fois sans être montré et aucune didascalie, ni même aucune remarque des personnages, n'attirent l'attention sur les costumes ou sur les décors.

12 La pièce s'ouvre sur une classique scène d'exposition entre le héros et son valet Arlequin, venus au Palais des Fées pour y trouver la Princesse dont le Prince est amoureux. Très tôt dans cette scène est livré un premier indice renvoyant le spectateur à l'histoire de Riquet à la houppe: "Fée implacable n'était-ce pas assez de me rendre affreux, fallait-il encore me donner le désir de plaire ${ }^{23}$ » s'apitoie le héros, auquel Arlequin répond pour atténuer son désespoir : « je ne vous trouve pas si laid que vous le 
dites; vous n'êtes pas beau à la vérité mais aussi n'êtes-vous pas horrible ${ }^{24}$ ». «Je dois le paraître à toutes celles que j'aimerai, c'est le supplice où m'a condamné la colère de la fée Bruyante ${ }^{25}$ " poursuit le Prince ${ }^{26}$. Quelques répliques plus loin, une nouvelle allusion au conte de Perrault renforce le dispositif : Arlequin se désole que son maître soit tombé amoureux d'une Princesse réputée aussi stupide que belle; le Prince lui répond: "La fée Agatine, pour adoucir le don fatal de la fée Bruyante, m'a doué secrètement du pouvoir de donner beaucoup d'esprit à celle que j'aimerais ${ }^{27}$ ".

Après une confrontation tendue entre le Prince, sa tortionnaire la fée Bruyante et Arlequin, qu'elle condamne à une faim éternelle pour lui avoir déplu, apparaissent la Princesse et son prétendant Lisandre, doté comme elle des agréments du corps et des avantages de la fortune, mais dépourvu d'esprit. Promise à ce beau benêt, la Princesse repousse les hommages respectueux du Prince lorsqu'elle le rencontre par l'entremise de la fée Agatine, tandis qu'Arlequin séduit malgré lui Sylvaine, la suivante de la Fée. Avant une nouvelle tentative d'attendrir sa belle, le Prince donne un peu d'esprit à celle-ci sur le conseil de la fée Agatine. La Princesse le reçoit moins mal. Elle écoute son histoire, qu'il lui présente sous la forme d'un conte de fées pour avouer son amour. Troublée, elle se montre compatissante en même temps qu'elle éprouve pour la première fois un agacement à l'égard de Lisandre, qu'elle doit épouser le soir même. Le Prince, toujours sur le conseil de la fée Agatine, double la dose d'esprit. La Princesse, reconnaissante de ce bienfait dont elle peut désormais juger tout le prix, lui promet de ne jamais épouser Lisandre. Pourtant, à l'issue d'une entrevue avec la fée Bruyante, elle semble être retombée dans sa bêtise première. Cette rechute est une victoire de la méchante Fée et désespère le Prince et sa protectrice. Mais coup de théâtre, la Princesse révèle qu'elle a feint « ce retour d'imbécillité ${ }^{28}$ " pour protéger le Prince de la colère de sa cruelle ennemie soupçonnant leur amour. Cet aveu porte la joie du Prince à son comble. La fée Bruyante arrive au moment où les amants s'apprêtent à quitter son Palais et menace de se venger. Tous deux préférant la mort à une séparation, l'Amour surgit pour les sauver et propose à la Princesse de transformer la figure de son amant. Elle refuse : «Prince [...] vous m'avez plu tel que vous êtes, et vous ne pouvez m'être plus cher que sous les traits que vous avez ${ }^{29} »$. Un divertissement, puis un vaudeville, roulant sur l'esprit et la beauté, annoncent les réjouissances prévues pour les deux hymens : celui du Prince et de la Princesse et celui de Sylvaine avec Arlequin, guéri de son appétit insatiable par l'Amour.

D'emblée, au travers de l'argument, on est frappé par la façon dont nos dramaturges ont choisi en même temps de se situer dans l'horizon d'attente suscité par le conte de Perrault et de s'en éloigner.

Premier critère : la liste des personnages. Si l'on retrouve bien les deux héros du conte, la Princesse (appelée Flore dans la pièce) et le Prince, on relève que ce dernier est anonyme, que l'œuvre ne comporte aucune référence au nom de Riquet à la houppe. Nos auteurs, par ailleurs, font l'économie de la Reine, mère de Riquet, ainsi que du Roi et de la Reine, parents de la Princesse, et de sa sœur cadette. Ils amènent au premier plan un rival du Prince, qu'ils nomment Lisandre.

Autre innovation: le dédoublement du personnage de la Fée en deux figures, l'une bienveillante, la fée Agatine, l'autre malfaisante, la fée Bruyante, dont le nom s'inscrit dans la tradition des patronymes comiques des contes merveilleux ${ }^{30}$. Enfin une domesticité qui n'existe pas chez Perrault tient ici une place importante: outre Sylvaine et Arlequin déjà cités, apparaît la suivante de la fée Bruyante, Alcine ${ }^{31}$. Une 
telle population de valets et de maîtres, ceux-ci pouvant avoir des liens de parenté avec les fées, effectifs (la fée Bruyante est la tante de Lisandre) ou suggérés (la fée Agatine se comporte comme si elle était celle du Prince), n'est pas sans évoquer la distribution d'une comédie conventionnelle, la condition royale des protagonistes n'étant jamais exploitée en tant que telle.

L'intrigue même ne renvoie-t-elle pas à une comédie amoureuse dans laquelle, en fin de compte, les fées ne joueraient qu'un rôle mineur? Romagnesi et Procope-Couteaux racontent-ils autre chose que les amours d'un jeune héros d'abord contrariées par une tante revêche et par la force des préjugés, ensuite servies par un auxiliaire dévoué et l'intelligence du cœur, pour voir finalement le jeune homme triompher de son rival et épouser celle qu'il aime, tandis que son valet épousera la suivante qui le courtisait? Le ressort de l'intrigue n'est-il pas tendu grâce à la métamorphose de l'héroïne qui, d'indifférente (pour ne pas dire hostile) aux avances du Prince, s'ouvrira graduellement à l'amour, en découvrant chemin faisant la conscience de soi, la compassion et la reconnaissance, avant d'affirmer avec force ses sentiments envers le Prince?

Si la manière de nos dramaturges n'exclut pas à l'occasion la bouffonnerie des lazzi d'Arlequin, ni une fantaisie digne des meilleures scènes foraines ${ }^{32}$, il n'en reste pas moins clair que cette féerie s'inscrit dans le sillage des comédies à succès de Delisle de la Drévetière, de Boissy et surtout de Marivaux : avec ce dernier particulièrement nos auteurs affichent une filiation évidente. Tant par sa forme que par son fond, la comédie des Fées se relie aussi bien à Arlequin poli par l'amour (créée chez les Italiens en 1720) qu'à La Seconde Surprise de l'amour (créée au Théâtre Français en 1727), chacune de ces deux pièces étant restée au répertoire et ayant été reprise au cours des mois précédant la création des Fées.

De même que Marivaux s'était imprégné du conte de Madame Durand Le Prodige d'amour pour jeter les bases d'Arlequin poli par l'amour ${ }^{33}$, nos dramaturges ont puisé dans un conte le motif de leur pièce. Et comme Marivaux, ils ont accompli un remarquable travail d'adaptation à partir du matériau d'origine, substituant le pouvoir de l'amour aux forces de la magie et aménageant des tensions dramatiques propres à maintenir en éveil l'intérêt du spectateur.

Ainsi ils introduisent une rivalité entre leur héros et Lisandre, le promis de la Princesse, de même qu'une rivalité entre la fée Bruyante et la fée Agatine, sources de pulsions contradictoires. De scène en scène, ils produisent des effets d'écho à répétition, jouant tantôt sur la symétrie des situations, tantôt sur leur opposition : le désir de conquête de la Princesse par le Prince, qui est au cœur du dispositif, tranche sur l'inertie de Lisandre, et se rapproche de l'obstination de Sylvaine à séduire Arlequin. Une autre mise en tension apporte une note comique, clairement doublée d'une résonance morale, entre l'insatisfaction amoureuse du Prince et l'insatiable voracité d'Arlequin, comme pour signifier qu'à chaque classe ses soucis. Ce à quoi renvoie peut-être la remarque de d'Origny qui mentionne en 1788 les "frustrations intéressantes » contenues dans cette «fable spirituelle » lorsqu'il commente la pièce dans ses Annales du Théâtre Italien ${ }^{34}$.

21 À l'auteur de La Surprise de l'amour nos dramaturges empruntent surtout son extraordinaire capacité à traduire par l'écriture les mouvements imperceptibles du cœur et de l'esprit qui animent les personnages, et en premier lieu la Princesse, dont le surprenant passage de la bêtise à l'intelligence (« l'esprit ») constitue la réelle gageure de la pièce. 


\section{agissante, d'une « parole-action » telle que l'a magistralement}

définie Michel Vinaver ${ }^{37}$ par opposition à une "parole instrument de l'action ${ }^{38}$ ", que Romagnesi et Procope-Couteaux font évoluer le

personnage de la Princesse d'un pôle, celui de la bêtise, à un autre, celui de l'esprit. Ils partent d'une situation initiale où Lisandre et elle sont conjointement soudés au pôle de la bêtise, tandis que le Prince, lui, est aimanté par celui de l'esprit ; pour en arriver à un dénouement où la Princesse a rejoint le Prince au pôle de l'esprit, tandis que Lisandre reste arrimé à celui de la bêtise, comme pour rendre la démonstration plus probante.

ressources $\mathrm{du}$ langage corporel - gestes, attitudes, mimiques pourtant traditionnellement intégrés au jeu des Italiens - pour traduire la trajectoire de l'héroïne. Dès les premières scènes, c'est dans le contenu du discours des personnages et dans la forme même de ces discours que l'action se produit. Examinons ce processus de près.

29 La bêtise de la Princesse et de Lisandre est d'abord évoquée à plusieurs reprises avant d'être (dé)montrée sur la scène. «Ce qui me fâche le plus pour vous, c'est que votre 
Maîtresse est d'une bêtise insupportable» (Les Fées, p.6) avoue Arlequin dès l'ouverture, avant de poursuivre : «qu'elle est belle disait l'un; oui, mais qu'elle est bête répondait l'autre» (Les Fées, p. 7). Son propos est relayé plus loin par la suivante Alcine, apostrophant la fée Bruyante au sujet de la Princesse et de Lisandre: "vous deviez bien, puisque vous vous intéressiez à leur destin, leur donner un peu d'esprit [...], vous oubliâtes le principal» (Les Fées, p. 16). La Fée se défend non sans cynisme : « du moins ne sont-ils à plaindre qu'à nos yeux, et leur extrême stupidité leur dérobe cette infortune » (idem).

Avec l'entrée du jeune couple, Acte I scène 5 (se tenant par la main comme deux enfants plutôt que comme le feraient deux amants), c'est la prise de parole de chacun, ou bien au contraire son aphasie plus éloquente encore, qui trahit en permanence les bornes de leur esprit : «à peine se disent-ils une parole en un quart-d'heure » (Les Fées, p.15) relève ironiquement Alcine, qui souligne plus tard: "Voyez comme ils répondent " pour stigmatiser le silence de la Princesse et de Lisandre à l'annonce de leur mariage.

31 Au long de cette scène et de la suivante, où les deux amants sont laissés en tête-à-tête, leur bêtise est signifiée par leur embarras à prendre la parole (ils n'ont jamais l'initiative de la conversation) ou par leur propension à esquiver les questions : «Oh dame, qu'elle parle la première » lance Lisandre à sa tante qui le presse d'exprimer ses sentiments. "Qu'elle commence toujours » répond-il encore devant l'insistance de la Fée. Des répliques courtes, une syntaxe élémentaire, maladroite et répétitive, un vocabulaire indigent, des mots eux-mêmes brefs voire monosyllabiques, caractérisent la conversation de ces deux niais :

BRUYANTE :

Ne le trouvez-vous pas aimable, bien fait?

LA PRINCESSE :

Oh! fort honnête.

ALCINE :

Vous ne répondez pas à ce qu'on vous demande. Le trouvez-vous beau, agréable?

LA PRINCESSE :

Pour cela oui.

ALCINE :

Et vous, Seigneur, comment trouvez-vous la Princesse?

LISANDRE :

Elle est bien jolie. (Les Fées, p. 19)

Outre ces particularités formelles, le couple affiche un infantilisme appuyé. Sa soumission à l'autorité de la fée Bruyante, figure inquisitrice qui le harcèle par ses interrogatoires, est totale ; à la question « ne serez-vous pas charmée d'avoir ce Prince pour époux ?» (p. 20), la princesse Flore répond platement et avec docilité : «Comme voudra ma tante ». Lisandre, lui, se plaint d'être grondé avant d'exécuter une révérence maladroite (celle du menuet et non celle en usage dans les règles de la civilité) quand la Fée lui ordonne: "parlez-lui donc, commencez par une révérence" (p.20). Les préoccupations exprimées sont par ailleurs plus propres aux enfants qu'à deux adultes sur le point de se marier : «c'est que j'aurais envie d'aller me promener dans le jardin ; tenez je chercherai des nids, j'en trouverai et je vous les apporterai » propose Lisandre qui s'ennuie déjà d'être avec sa promise (p. 24). Scène 7, la Princesse se réjouit en enfant charmée (elle "rit" précise une didascalie exceptionnelle) des acrobaties qu'Arlequin accomplit autour d'elle à la manière d'un petit chien barbet: " qu'il est plaisant ! », « il est tout à fait divertissant » (p. 29-30). Enfin le narcissisme de Lisandre 
- «Je suis beau n'est-ce pas? [...] je le sais il y a longtemps; à la Cour de mon père toutes les Dames, quand elles me voyaient, disaient, ah qu'il est beau, ah qu'il est beau ! et moi je riais, car j'étais bien aise » (p. 23) - confine au burlesque, de même que la fière assurance de la Princesse qui se vante de ne pas avoir peur de rester seule «quand il fait jour » (idem p. 23).

Dotés d'un langage et d'un comportement puérils, enfermés dans une bulle qui les coupe du monde (le château de la fée Bruyante), nos héros sont également coupés d'eux-mêmes, incapables d'envisager leurs propres sentiments. La vue, qui leur livre la surface des choses et des êtres, est leur seul mode d'accès à l'autre : «Vous me regardez bien? » s'étonne Lisandre ; «C'est que cela me fait plaisir » se justifie la Princesse en guise de déclaration d'amour. Partant, ils sont impuissants à s'affranchir du champ du matériel pour atteindre celui de l'abstraction: «Moi, je ne sais pas ce que je pense » avoue ingénument Lisandre avant de s'emporter : «Oh! je ne saurais deviner ce que j'ai à lui dire!» (Les Fées, p. 19 et 21). Dans cette perspective toujours, ils ne peuvent s'affranchir de l'instant présent; lorsqu'ils se projettent dans le futur, c'est pour substituer un jeu à une réalité, dans ce cas le mariage qui les attend : " prenez un nid de pies, nous les élèverons " s'enthousiasme Flore ; «Et nous leur apprendrons à parler " enchaîne Lisandre, dans l'inconscience de la charge ironique de sa réflexion!

Cette opacité - qui n'est pas celle de l'enfance comme nos auteurs tendent à le faire croire mais plutôt celle d'un degré zéro de l'humanité - s'accompagne d'une rusticité, voire d'une grossièreté dont les exemples se multiplient à l'envi : «Ah qu'il est laid !» (Les Fées, p. 25) s'exclame tout haut la Princesse la première fois qu'elle est présentée au Prince. «Il ne m'est pas possible de le regarder » répète-t-elle lorsque la fée Agatine lui enjoint de «[faire] un effort, la bienséance l'exige » (p. 26). Sourde à cette voix, la jeune femme persiste: "Vous me verrez tant qu'il vous plaira pourvu que je ne vous voie point » (p. 28), une réponse qui amène Agatine à la morigéner : «Sont-ce là les égards que vous devez à une personne de votre rang?» (idem). Autre manifestation de la brutalité de la jeune femme quand le Prince lui fait présent d'Arlequin (épisode d'une étonnante cruauté) pour la divertir par ses tours de souplesse : «Oh je ne veux rien de vous!» (p. 31) s'exclame-t-elle sans ménagement, tandis que la Fée la sermonne une nouvelle fois : « Vous auriez pu le refuser plus poliment » (idem).

Pourquoi une telle insistance sur ce trait de caractère de l'héroïne? Sans doute pour mieux marquer la distance qui la sépare du pôle de l'esprit, et donc du Prince, dont le raffinement de langage, de pensées, de sentiments et de manières lui sont parfaitement étrangers.

36 Car le discours de ce héros, comme par l'effet d'un miroir inversé courant dans les contes merveilleux, s'oppose en tout point au discours des ignorants et des sots que sont la Princesse et Lisandre. Par la forme aussi bien que par le fond, là encore, sa conversation manifeste toutes les qualités d'un homme d'esprit.

Par la forme d'abord. Le Prince s'exprime avec aisance, ses répliques sont longues, syntaxiquement élaborées; des champs lexicaux fournis lui permettent d'exprimer les nuances de sa pensée et de ses sentiments; son style est élégant, parfois précieux ("Charmante Princesse », « divine Princesse », « adorable Princesse », Les Fées, p. 25, 27 et 31), et touche au tragique lorsqu'il évoque sa "destinée ", "le supplice» où l'a condamné la colère de sa « mortelle ennemie », « le don fatal » qu'elle lui a fait, « l'état effroyable " où le réduit le charme qu'elle a répandu sur lui... Jusqu'à accéder aux accents d'un héros racinien : «la raison m'exile de ces lieux, l'amour m'y retient, et de 
quelque côté que je me détermine, le désespoir ou l'absence me mettra au tombeau » (p. 42).

La scène entière de la première rencontre entre le Prince et la Princesse (acte I scène 7) est comme traversée de courts-circuits provoqués par l'alternance de la parole déliée et policée emblématique de la maturité de l'un, avec la parole fruste trahissant la puérilité de l'autre :

LE PRINCE :

Ah! Madame, qu'on est malheureux de ressentir tant d'amour lorsqu'on est sûr de déplaire ; je n'ose vous découvrir des sentiments dignes de la beauté qui me les inspire, et qui feraient son bonheur et le mien, sans l'obstacle que ma laideur oppose à mon amour.

LA PRINCESSE :

Ce n'est pas ma faute, Seigneur (Les Fées, p. 26).

Échange qu'Arlequin commente sur un ton désabusé : « Mon pauvre Maître, autant de rhétorique perdue » (p. 26).

Pour ce qui est du fond, on relève que la maîtrise du langage dont fait preuve le Prince va de pair avec son aptitude à sonder son cœur et celui de ses interlocuteurs, à exposer ses désirs et à les défendre, à s'opposer enfin à la volonté d'autrui quand elle est contraire à ses vues : fort de son éloquence, le Prince successivement brave l'autorité de la fée Bruyante (Acte I scène 2) et affronte avec courage les dédains de la Princesse (acte I scène 7).

1 Renonçant à tout accessoire enchanté (baguette, anneau, ceinture), nos auteurs prennent le parti de montrer degré par degré, au cours de cinq entretiens qu'elle a avec le Prince ${ }^{41}$, la lente accession de l'héroïne à la conscience d'elle-même et son ouverture à l'amour. Et plutôt que l'effet supposé des deux interventions magiques du Prince ("Hé bien je n'ai qu'à lui donner de l'esprit, grâce à vos bontés, j'en ai le pouvoir » (Les Fées, p. 45) se souvient-il Acte II scène 1; «Redoublez la dose d'esprit » lui conseille Agatine, acte II scène 4), c'est en réalité le pouvoir des mots, quand ils sont inspirés par l'amour ou par la bienveillance, que nos dramaturges mettent en scène.

Le premier signe de l'amorce de la métamorphose de la princesse Flore est l'air rêveur qu'observe chez elle la fée Agatine au début de la scène 2, acte II. Ce signe est aussitôt accompagné par un aveu révélateur du changement, même si la formulation demeure enfantine: «Je viens de me souvenir de quelque chose qui me fâche; j'ai peur d'avoir mal parlé tantôt, et d'avoir fait de la peine à quelqu'un » (Les Fées, p. 47). Déjà la jeune femme est capable de s'extraire du temps présent pour réfléchir à une action passée, d'extérioriser un sentiment et d'entrevoir le pouvoir d'une parole agissante, qui peut faire mal à autrui. Plus loin elle « [se] rappelle » le discours du Prince et, ô stupeur, elle note qu'il «était très bien tourné » (p. 48), avant d'exprimer un désir au conditionnel et de pénétrer, de fait, dans l'espace de la sociabilité des adultes : «Je voudrais lui faire des excuses » (idem) dit-elle parlant du Prince.

3 Second signe fort de changement, la Princesse refuse désormais d'être divertie par les culbutes d'Arlequin : «Non, elles ne seraient plus de mon goût » (p. 49) reconnait-elle, comme pour mieux marquer la rupture avec son état antérieur.

Prenant toujours plus d'assurance au cours de ce troisième entretien ${ }^{42}$, Flore ponctue de ses interventions le conte que lui raconte le Prince, donnant diverses preuves de son intelligence et de sa sensibilité nouvelles: "Ah ! que ce Prince avait de délicatesse ", «Je le crois bien », «Ah! c'était le Prince Hibou, je gage ». Elle prend même l'initiative 
de poser des questions : «Et quel est ce remède ? ", « Elle l'aima, sans doute ? (Les Fées, p. 51-53), elle qui autrefois ne prenait la parole que contrainte et forcée. La longueur et la formulation de sa dernière réplique dans la scène : «Les malheureux ont un grand ascendant sur les cœurs compatissants, et je crois que je ne saurais refuser la pitié la plus tendre à un Prince que j'aurais mis en cet état » (p. 53) révèle un rapprochement avec l'exquise courtoisie du Prince et quelque ressemblance avec son élocution aisée.

Acte II scène 3, la rencontre entre la Princesse, le Prince, son rival Lisandre, la fée Agatine et Arlequin semble ne pas avoir d'autre fonction que de montrer plus clairement encore le chemin parcouru par Flore en direction de l'esprit, tandis que Lisandre manifeste, en repoussoir, tous les travers de leur bêtise autrefois commune. "Me voilà » claironne-t-il, satisfait de lui-même, alors qu'il revient de son escapade de dénicheur de pies. L'échange qui s'en suit avec le Prince illustre la profonde opposition entre les deux prétendants :

LE PRINCE :

Seigneur, c'est un Prince qui est épris des beautés de la charmante Flore et qui vous la disputerait au péril de votre vie ou de la sienne, s'il ne respectait, jusque dans son rival même, la Maîtresse qu'il adore.

LISANDRE :

Ah! ah! ma tante m'a parlé de vous. Elle m'a dit que vous étiez amoureux de ma femme, mais que je n'avais rien à craindre. (Les Fées, p. 55)

Toujours maladroit et insensible, Lisandre répond à côté quand la Princesse l'interroge : «Avez-vous pensé à moi ?» (p. 55). Il s'emporte contre le Prince : « Hé de quoi se mêle-t-il donc? Est-ce que cela le regarde?» (p.56) et sur un ton d'enfant capricieux, il menace: "C'est que je n'aime pas qu'on m'obstine moi, et je vais me plaindre à ma tante » et plus loin encore : « je m'en vais aussi me plaindre de vous tout d'un temps ; là !» (p. 57).

Témoin à la fois agacé par ces marques d'indélicatesse et touché par la compréhension magnanime du Prince, la Princesse découvre simultanément son amant et le Prince sous un jour nouveau: "Il me paraît bien borné " dit-elle du premier à Agatine; «Lorsqu'il parle, il fait presque oublier qu'on le voit» dit-elle du second avant de se demander : «Pourquoi Lisandre n'a-t-il pas l'esprit de l'autre? », réflexion révélatrice de l'entendement qui est désormais le sien...

Parce qu'elle est sortie de son état de débilité mentale et qu'elle maitrise désormais le langage (elle ne dit plus « j'ai beaucoup de chagrin de vous voir comme cela » (Les Fées, p.27) mais «Je suis au désespoir de l'avoir vu, puisque je suis la cause de son infortune » [p.60]), Flore accède à la découverte d'elle-même et désire connaître la genèse de ce bouleversement. Dans le sillage de la pensée des philosophes qui se sont interrogés après John Locke sur l'histoire de l'esprit humain ${ }^{43}$, elle s'étonne :

ne vous apercevez-vous pas du changement qui s'est fait en moi depuis quelques moments? je pense, je m'exprime. Il me semble que ce n'est que d'aujourd'hui que je commence à vivre. Mon esprit n'était jusqu'ici qu'un chaos, les idées s'y débrouillent, s'y éclaircissent ; d'où peut naître cette nouveauté ? (Les Fées, p. 61)

Donnant raison à Agatine, qui soutient que «l'esprit développe et remue les passions » (45), que « l'esprit fait naître les sentiments » (p. 60), la Princesse s'humanise au fur et à mesure qu'elle s'éloigne de son état premier, sous la houlette vigilante du Prince.

Observons cette autre trajectoire. Sincère, sans oublier d'être fin psychologue, le Prince prétend dans un premier temps ne solliciter que «la pitié » de la jeune femme, sa "générosité au défaut de [sa] tendresse »: "dites-moi seulement que vous me 
plaignez "(Les Fées, p. 27), "cette seule compassion me suffit»(p. 28). Après la déclaration qu'elle reçoit du Prince, sans pouvoir y répondre en raison de l'arrivée intempestive de Lisandre, Flore en appelle à la compassion de celui-ci pour le Prince : " soyez généreux; c'est bien assez pour ce Prince d'être si mal traité de la nature et de l'amour » (p. 57). Dans la scène suivante (acte II, scène 4), le Prince rebondit sur ce mot de "générosité " que la Princesse associe spontanément aux mots de « reconnaissance » et d'« estime ». Plus loin (acte II scène 5), c'est toujours elle qui opère un glissement de la "pitié sincère » à " la tendresse » qu'elle dit ne pas pouvoir avoir pour lui. Après les menaces de la fée Bruyante (acte II scène 8), elle tient à lui donner " une preuve de sa plus vive reconnaissance [...] et l'assurer que si [elle] ne l'aime pas, [elle] voudrai[t] l'aimer, du moins » (p. 66-67); puis elle explique à Agatine que la fée Bruyante « a fait naître [...] dans [s]on cœur la pitié la plus sensible » pour ce «Prince trop généreux » dont elle se désespère d'être la source de tous les malheurs. C'est directement au Prince, dans la scène suivante, qu'elle s'adressera en héroïne tragique, à son tour, l'assurant dans une longue tirade d'abord toujours de sa « reconnaissance » et de sa " plus tendre estime », avant d'enchaîner par une série de déclarations de moins en moins équivoques:

Je vous jure de ne faire jamais de vœux que pour vous; que toutes mes pensées, que toutes mes actions n'auront jamais en vue que mon généreux bienfaiteur.

[...]

Prince, je veux que vous me promettiez de vivre, que vous me le juriez par moimême, ou j'atteste ici le Ciel, que votre mort sera suivie de la mienne.

$[\ldots .$.

Vous dirai-je plus? ne craignez aucun rival, je renonce pour jamais au joug de l'hymenée; mon cœur est rempli des sentiments qu'il a pour vous, il n'en peut souffrir d'autres ». (Les Fées, p. 70, 71, 72)

51 Ses propos appellent un commentaire de la fée Agatine, qu'on s'attendrait plutôt à trouver dans la bouche d'Arlequin: «Je crois qu'elle aime.» (p.72). Le geste de la Princesse, offrant sa main à baiser au Prince, conforte l'hypothèse. "L'amour a parlé sous le nom de la reconnaissance; j'ai vu de véritables inquiétudes, j'ai entendu des soupirs, je n'en doute presque plus, Flore est sensible ( (Les Fées, p. 74) poursuit Agatine, jouant plus que jamais en fait le rôle de la suivante perspicace d'une nouvelle Surprise de l'amour. Acte III scène 7, la Princesse sera enfin en mesure d'exprimer ses sentiments : «Oui, Prince, je vous aime, et ne rougis point de vous le dire; mon amour est d'autant plus fort, qu'il a vaincu tous les préjugés » (p. 98). Quel chemin accompli depuis le premier mouvement de répulsion qui s'est produit quelques heures à peine auparavant !

52 Se serait-elle achevée à la fin du second acte, la pièce de Romagnesi et ProcopeCouteaux aurait pleinement rempli son programme en tant que transposition de Riquet à la houppe au théâtre. Pourtant nos dramaturges ne s'en sont pas tenus là : ils ont ajouté un développement inattendu à leur argument, avec un troisième acte et un coup de théâtre qui est pour beaucoup dans le succès de l'œuvre ${ }^{44}$.

53 À la scène 3 de l'acte III, alors que l'amour de Flore pour le Prince est avéré, survient l'impensable: la fée Bruyante annonce à la cantonade que la Princesse épouse son neveu Lisandre « dans une heure ». Les trois scènes suivantes confirment l'information et donnent à penser à tous que la jeune femme est retombée dans son état de bêtise originel. De fait, la belle éloquence de Flore s'est envolée. Ses répliques, courtes, sèches et 
impersonnelles, renvoient à ses difficultés premières d'expression: "Assurément.", "Je l'ai bien vu », "Oui », " Vous aviez raison", « Non vraiment », «Cela ne serait pas bien », «Sans doute » (Les Fées, p. 94-95) sont les seules paroles que Lisandre parvient à lui arracher scène 6. Les propos de la Princesse sont de nouveau incivils et offensants : "Ah! voilà encore ce Prince ; vous disiez que vous le feriez sortir de votre Palais?" (p. 90). Leur inconvenance ingénue rappelle aussi les incongruités de Lisandre: « Mais si Lisandre ne vient point, je l'irai chercher, moi [...] Il y a donc du mal à chercher son mari?» (p.91). Enfin une syntaxe chaotique trahit l'infantilisme affectif qui la caractérisait lors de son premier entretien avec le Prince : à la question «pourquoi tantôt demandiez-vous qu'on retardât ce mariage? » posée par la fée Bruyante, Flore répond «À cause que Lisandre m'avait grondée; mais je lui pardonne, parce que je l'aime bien » (idem).

Les signes de cette régression qui s'accumulent ainsi au niveau verbal sont indicatifs pour tout le monde (le public compris) de sa rechute et sont propres à calmer les inquiétudes de la Fée («son ingénuité me charme» (Les Fées, p.91) dit-elle avec satisfaction) qui s'était alarmée, acte II scène 6, de la résistance inattendue que lui avait opposée la Princesse à l'évocation de son très prochain mariage avec Lisandre. Furieuse de voir son autorité contestée, elle avait réagi vivement: "Vous ne faisiez point ces questions-là tantôt », avant d'ajouter : « Obéissez, vous dis-je » (p. 64) et de proférer des menaces contre le Prince.

Devenue réceptive et douée d'une perspicacité nouvelle exacerbée par ses sentiments naissants, Flore a subodoré le danger qui pesait sur le Prince à partir de ce moment-là et deviné que le seul moyen de l'en protéger était, pour lui donner le temps de s'éloigner du Palais, de faire croire à Bruyante qu'elle était toujours la créature malléable que la Fée tenait sous sa coupe. Parce qu'elle a compris, en quelque sorte, que son accès au savoir avait été conditionné par sa maîtrise du langage, et que la Fée, de son côté, avait assimilé le déploiement de sa parole à son déniaisement, Flore s'impose un retour à une langue fruste, au service d'une pensée fruste, pour détourner les soupçons de sa nouvelle ennemie. Outre Bruyante, la Princesse trompe Lisandre que la Fée a affecté à sa garde : «Ah! bon, vous parlez comme moi à présent, car tantôt je ne vous entendais pas, et vous aviez un air éveillé que je n'aimais pas non plus » (Les Fées, p. 95).

56 Cette feinte, par laquelle elle anticipe les manœuvres de Bruyante et les déjoue, est la preuve éclatante de son passage du côté du pôle de l'esprit. L'élève a même surpassé son maître puisque son artifice n'a été soupçonné ni par la fée Agatine, ni par le Prince, pourtant tous deux d'une intelligence hors du commun. Ce qui d'ailleurs étonne la Princesse, tant sa foi - comme celle de tous les nouveaux convertis - dans leur génie est grande :

est-il possible que vous ayez été abusé comme les autres ? Se peut-il que celui de qui j'ai appris à penser et à sentir, se reconnaisse si peu aux mouvements du cœur et de l'esprit? ne vous êtes-vous pas aperçu que ce retour d'imbécillité était un effet de ma tendresse? (Les Fées, p. 97)

Quant au spectateur, pas plus clairvoyant que le Prince et la Fée d'après les témoignages contemporains, il avait au moins, lui, un indice pour flairer la manœuvre : au cours du dernier tête-à-tête entre le neveu de Bruyante et la Princesse, tandis que Lisandre se réjouit en enfant gâté des « mille belles choses, des bals, des feux d'artifice, des tournois » que sa tante fera organiser pour les divertir à leurs noces, Flore glisse avec malice que « le meilleur » a été oublié, l'incitant, pour l'éloigner, à s'en entretenir 
avec la Fée. Or qu'est ce «meilleur» auquel songe la Princesse? Un opéra : «Je me meurs d'envie de voir un opéra, on dit que cela est si beau». Loin de percevoir l'incongruité d'un souhait aussi raffiné dans la bouche de sa promise, Lisandre s'en va tout guilleret, avec l'idée de demander du même coup pour lui « des marionnettes »! (Les Fées, p. 96)

58 Au fil de notre lecture, la pièce de Romagnesi et Procope-Couteaux n'a cessé de nous surprendre par l'espace de réflexion qu'elle ouvre, auquel son titre, Les Fées, ne permettait pas de s'attendre. Elle nous a surpris d'abord par la distance qu'elle a prise avec le conte de Perrault, distance que la critique a entérinée à sa façon en "ignorant » cette filiation, se contentant de signaler que la comédie avait pour sujet l'Esprit préférable à la beauté, maxime empruntée à la sagesse des nations... Faut-il en déduire que le conte de Perrault n'était plus une référence à l'époque où paraissent les notices consacrées à la pièce (à partir de 1763 avec le Dictionnaire des théâtres de Léris ${ }^{45}$ ) ?

Autre sujet d'étonnement : à aucun moment n'est décrite dans Les Fées la disgrâce physique du héros. Nos dramaturges répètent qu'il est " affreux ", « laid », « horrible », « désagréable » mais pas une didascalie ni le moindre discours ne brosse le portrait du Prince. Perrault au contraire se répandait sur les tares du " petit homme», soulignant " la difformité de son corps ", " la laideur de son visage ", " sa bosse », sa claudication, son strabisme, "son gros nez rouge ", le définissant dès la première phrase, rappelonsle, comme «si laid et si mal fait, qu'on douta longtemps s'il avait forme humaine ${ }^{46}$ ». Pourquoi cette ellipse dans la pièce ? Il n'est pas interdit de penser qu'elle se relie au fait que Procope-Couteaux lui-même " était contrefait [entendez difforme, bossu], laid et noir, au point qu'on a dit qu'il suait de l'encre" comme le rapporte sans ménagement Antoine de Léris dans la notice biographique de l'auteur ${ }^{47}$. Comment ne pas être fasciné par ce télescopage entre réalité et fiction? Télescopage d'autant plus déflagrant que le personnage du Prince, comme le révèle le Mercure de France dans son compte rendu, était interprété par le co-auteur de la pièce, Romagnesi lui-même ${ }^{48} .$.

60 Au sujet de Procope, Léris note encore qu'il « était docteur en médecine de la Faculté de Paris; il avait très bien étudié, et avait beaucoup d'esprit et d'enjouement ${ }^{49}$ ». Né en 1684 et mort en 1753, c'était un homme cultivé de la première moitié du XVIII siècle, franc-maçon de la première heure, ouvert à la science et, à sa manière, philosophe : une foi inconditionnelle dans l'entendement humain, l'optimisme triomphant de l'intrigue bipolaire qu'il compose avec Romagnesi l'attestent sans détour. D'un côté sont mis en scène l'obscurantisme, la Bêtise, la Soumission et la Tyrannie; de l'autre, l'Amour et la Raison pacifiés, la Conscience de soi et l'Ouverture au monde. Nous ne sommes pas loin d'une allégorie. Et celle-ci, par personnages de comédie interposés, délivre un message à valeur universelle fondé sur la primauté de l'expérience au sens où l'entend Locke, dont la tonalité militante nous paraît pour l'époque d'une extrême modernité. Écoutons la fée Agatine, parlant de la Princesse :

Bruyante n'a plus affaire à une personne bornée qui craint les menaces, ou se laisse séduire par les promesses: c'est Flore éclairée [c'est nous qui soulignons], une Princesse qui connait, qui ressent, qui s'exprime, qui lui opposera son antipathie pour son neveu, les raisons qu'elle a de le refuser, et qui fera valoir les droits qu'une Princesse libre doit avoir sur elle-même. (Les Fées, p. 84)

61 En même temps qu'ils invitent, à leur manière, le spectateur à assister au " triomphe de l'amour ", « au préjugé vaincu » et à un « heureux stratagème ", Romagnesi et ProcopeCouteaux le convient à une réflexion rien moins qu'idéologique, qui connaîtra une résurgence en 1744 avec La Dispute de Marivaux ${ }^{50}$. Plutôt que de nous attarder sur le 
réseau de correspondances qui relient La Dispute aux Fées (dont Marivaux ne pouvait ignorer l'existence), nous préférons souligner, pour l'heure, la place que mérite notre féerie - aujourd'hui complètement oubliée - dans l'histoire de la pensée française du $\mathrm{xVIII}^{\mathrm{e}}$ siècle. En raison de son argument novateur aussi bien que de la fonction qu'elle a $\mathrm{pu}$ occuper dans la formation de l'esprit des spectateurs sur près de vingt ans, entre 1736 et 1754 .

Car les scènes parisiennes sont alors de vrais laboratoires où les dramaturges fixent les idées du temps. Représentée huit ans avant La Dispute de Marivaux, la comédie des Fées se trouve avoir été créée un an et demi après l'opéra-comique de Pannard et Laffichard Pygmalion, d'abord joué à la Foire Saint-Germain 1735, puis repris en... 1736 et dans une nouvelle version du seul Pannard en... 1744. Nous avions souligné, il y a quelques années, cette proximité chronologique et thématique de la comédie de Marivaux avec ce Pygmalion, puis montré comment la pièce de la Foire abordait sur le mode ironique des idées que Condillac, Diderot, Rousseau ou Buffon développeront plus tard dans leurs travaux ${ }^{51}$. Le 12 janvier 1741 Romagnesi et Procope-Couteaux (à partir de l'idée d'un dénommé Baurans) deviendront à leur tour, au Théâtre Italien, les auteurs d'une comédie en trois actes intitulée... Pygmalion. Rappelons qu'en amont, les Lettres philosophiques de Voltaire (dont la XIII eù Voltaire affirme son adhésion à la pensée de Locke) ont connu un succès éclatant dès leur publication en 1734, succès renforcé par le scandale lié à leur interdiction. Deux ans plus tard, en 1736, dans une "Épître à Madame la Marquise du Châtelet " publiée en tête de sa tragédie Alzire (créée à la Comédie-Française le 27 janvier de la même année ${ }^{52}$ ), Voltaire évoque "l'Esprit philosophique [qui] fait tant de progrès en France depuis quarante ans ", non sans avoir cité auparavant le nom de Locke, " ce sage précepteur du genre humain ${ }^{53}$ ".

Cette série réunissant sur une dizaine d'années les Pygmalion de la Foire et des Italiens, Les Fées des Italiens et La Dispute de Marivaux à la Comédie-Française est d'une exceptionnelle densité, qui déborde largement l'histoire du théâtre ${ }^{54}$. Elle nous conduit à penser que d'une part, la porosité des œuvres dramatiques entre elles et, d'autre part, leur imprégnation par d'autres productions de l'esprit - dans ce cas, celles qui contribuent à la propagation de l'empirisme - sont des facteurs à ne pas négliger si l'on désire appréhender l'aube des Lumières dans l'infinie variété de ses manifestations.

\section{NOTES}

1.Précisément 508 spectateurs payants selon Clarence D. Brenner (The Théâtre Italien, its repertory, 1716-1793, Berkeley and Los Angeles, University of California Press, 1961, coll. "University of California Publications in Modern Philology"; 63).

2.Actuellement à la hateur du 29 rue Etienne Marcel à la limited u 2e arrondissement à Paris.

3.L'absence de registres à la Comédie-Italienne pour 1738 et 1739 explique qu'aucune reprise ne soit signalée au cours de ces années-là. 
4.La comédie Le Conte de Fées eut onze representations lors de sa creation en mai 1735 et n'est reprise qu'une fois en 1736 pour deux representations (le 1er et le 3 janvier). La comédie Les Fées rivales est représentée dix-sept fois à partir du 19 septembre 1748 et ne sera reprise qu'une fois pour six representations en janvier 1749.

5.La première attira le nombre prodigieux de 1270 spectateurs le jour de sa creation, la seconde 876 spectateurs, d'après Brenner toujours.

6. Chez le Breton à Paris pour ces deux editions in- $8^{\circ}$ (de respectivement 80 p. et 64 p.). 7.Notre édition de référence pour la présente étude (Le Nouveau Théâtre Italien ou Recueil général des comédies représentées par les Comédiens Italiens Ordinaires du Roi, nouv. éd., Paris, Briasson, 1753, t. 9, pagination séparée).

8.On peut lire dans le compte rendu que lui consacre le Mercure de France en août 1736 (p. 1882-1891) : «Cette comédie a été reçue du public avec un applaudissement général ». Desboulmiers notera dans son Histoire anecdotique et raisonnée du Théâtre Italien depuis son rétablissement en France jusqu'à l'année 1769 : « La fable de cette pièce fut trouvée très agréable, quelques situations très intéressantes et l'ensemble fut très applaudi » (Paris, Lacombe, 1769, t. 4, p. 257).

9.Opéra-comique en trois actes de Fuzelier, représenté à la Foire Saint-Laurent 1732 (BnF, Département des manuscrits occidentaux, fonds français (ci-après BnF f. fr.), «Théâtre inédit de Fuzelier », 9337).

10.Opéra-comique en un acte de De La Grange, représenté à la Foire Saint-Gemain 1734 (BnF f. fr. "Théâtre inédit de la Foire ", 9313).

11.Pièce en un acte de d'Allainval, représentée à la Foire Saint-Laurent 1734 (BnF f. fr., "Théâtre inédit de la Foire ", 9313).

12.Pièce d'un acte d'un anonyme représentée à la Foire Saint-Laurent 1735 (BnF f. fr., "Théâtre inédit de la Foire ", 9313).

13.Prologue de Charles-François Pannard, représenté à la Foire Saint-Laurent 1736 (BnF f. fr., " Théâtre inédit de Pannard ", 9323).

14.Pièce d'un acte d'un anonyme représentée à la Foire Saint-Germain 1737 (BnF f. fr., "Théâtre inédit de la Foire ", 9313).

15.Opéra-comique en un acte de Carolet, représenté à la Foire Saint-Laurent 1737 (BnF f. fr., " Théâtre inédit de Carolet », 9315).

16. Nous nous permettons de renvoyer le lecteur à notre édition critique de ces deux pièces dans le volume 5 de la série «Bibliothèque des Génies et des Fées » dirigée par Nadine Jasmin (Paris, Honoré Champion, 2007, coll. « Sources classiques »).

17.Seule la pièce de Dancourt sera rejouée en 1753-1754.

18. Rappelons qu'une fée justicière, dans cette fable, agrémentait les paroles d'une sœur cadette de roses, de perles et de diamants pour la récompenser de sa serviabilité, tandis qu'elle condamnait la sœur aînée à cracher des vipères et des crapauds pour la punir de son orgueil et de son arrogance.

19.Nous citerons l'édition critique de Tony Gheeraert dans Perrault, Fénelon, Mailly, Préchac, Choisy et anonymes. Contes merveilleux, « Bibliothèque des Génies et des Fées » (ouvr. cité, 2005, t. 4, p. 233).

20. Charles Perrault, Riquet à la houppe, ouvr. cité, p. 234.

21.Ibid., p. 238.

22.Ibid., p. 236.

23.J.-A. Romagnesi et M. Procope-Couteaux, Les Fées, ouvr. cité, p. 5.

24.Idem.

25.Idem. 
26. Cette colère de la fée Bruyante, qui n'a pas été conviée à la noce de la Reine dont elle avait pourtant servi les amours, ne figure pas dans Riquet à la houppex; elle rappelle en revanche la malédiction de la vieille Fée qui n'a pas été invitée au baptême de la Princesse dans La Belle au Bois Dormant.

27.Les Fées, ouvr. cité, p. 7.

28.Ibid., p. 97.

29.Ibid., p. 108.

30.Une fée Violente œuvre dans La Chatte Blanche de $\mathrm{M}^{\text {me }} \mathrm{d}^{\prime}$ Aulnoy ; les fées Cruelle et Envieuse apparaissent dans La Tyrannie des fées détruite de $\mathrm{M}^{\text {me }}$ d'Auneuil. En 1735 Romagnesi et Riccoboni mettent en scène une fée Rancunière dans la comédie Le Conte de fées.

31.Ce nom d'Alcine est-il emprunté à la magicienne, sœur de la fée Morgane, dans le Roland furieux de l'Ariostex?

32. Citons les deux divertissements aviaires orchestrés par Arlequin à la fin du premier et du second acte. Dans le premier épisode Arlequin, privé des nourritures terrestres mais doté du pouvoir de se nourrir du chant des oiseaux, convoque tour à tour un rossignol, un serin de Canarie, une alouette et une caille qui lui offrent une sérénade en guise de repas. Par un effet de miroir inattendu, Arlequin est privé à son insu de son pouvoir magique dans le second épisode et déclenche, au lieu d'une sérénade, le braiment d'un âne, le cri d'un cochon, l'aboiement d'un chien et le miaulement d'un chat quand il enjoint aux oiseaux de le rassasier. Cette cacophonie le met en fuite sans autre prolongement dans l'intrigue. On relève que ces deux épisodes parallèles font suite respectivement à deux scènes comiques de la pièce, des duos entre Arlequin (valet du Prince) et Sylvaine (la suivante de la fée Agatine) qui le poursuit de ses assiduités (acte I, scène 10 et acte II, scène 11).

33. Nous renvoyons le lecteur à l'édition critique de ce conte par Raymonde Robert dans la Bibliothèque des Génies et des Fées (ouvr. cité, 2005, t. 2, p. 475-495). Pour l'identification de ce conte en tant que source de la comédie de Marivaux, voir les travaux de Shirley Jones (" A probable source of Marivaux's Arlequin poli par l'amour », French Studies, 1965, vol. XIX, p. 385-391) et l'introduction de la pièce par Frédéric Deloffre (Théâtre complet de Marivaux, éd. établie par Frédéric Deloffre et Françoise Rubellin, Paris, Classiques Garnier multimédia, 2000, coll. « Classiques Garnier »).

34.Antoine-Jean-Baptiste-Abraham d'Origny, Annales du Théâtre Italien depuis son origine jusqu'à ce jour [...], Paris, Vve Duchesne, 1788, t. 1, p. 146.

35. Charles Perrault, Riquet à la houppe, ouvr. cité, p. 235. (C'est nous qui soulignons).

36.Idem.

37.Dans « Méthode d'approche du texte théâtral ", Écritures dramatiques. Essais d'analyse de textes de théâtre (Arles, Actes Sud, 1993, coll. " Répliques », p. 891-911).

38.On remarque que pour Michel Vinaver l'écriture de Marivaux est précisément caractérisée par la force singulière d'une parole-action quand l'écriture d'un Beaumarchais, au contraire, se caractérise généralement par une « parole instrument de l'action ».

39.Jean-Antoine Romagnesi et Michel Procope-Couteaux, Les Fées, ouvr. cité, p. 64.

40.Nous citons le Dictionnaire portatif, historique et littéraire de Léris $x$ : « Le sujet de [cette comédie], qui fut applaudie, et qu'on rejoue de temps en temps, est l'Esprit préférable à la beauté ». (Paris, C. A. Jombert, 1763, p. 187).

41.Le premier acte I scène 7 , le second hors champ, le troisième acte II scène 2, le quatrième acte II toujours, scène 9 et le dernier acte III scène 7. 
42.Rappelons que leur première entrevue fut marquée par la conduite puérile et grossière de Florex; la seconde s'est déroulée hors champ, mais le Prince confie à Agatinex: « Je lui ai inspiré la même horreur qu'à la première ». (Les Fées, p. 42). 43.Voir l'ouvrage fondamental de Jean Dagen L'Histoire de l'esprit humain dans la pensée française de Fontenelle à Condorcet, Paris, Klincksieck, 1977 et Lille, Atelier de reproduction des thèses de l'Université Lille 3, 1980

44.« L'objet principal de la pièce paraissait rempli, puisque l'esprit l'avait emporté dans son cœur sur les charmes de la beautéx; mais les auteurs ont réveillé la curiosité par un coup de théâtre, qui d'abord a fait prendre le change aux spectateurs ». (Desboulmiers, ouvr. cité, t. 4, p. 255).

45.Le compte rendu du Mercure de France d'août 1736 déjà cité ne mentionne pas plus Riquet à la houppe que Perrault (comme si ces références allaient de soi alors), mais l'auteur y assimile une première fois la comédie à un « conte de fées " et rappelle à la fin que son " sujet est tiré d'un conte ».

46.Charles Perrault, Riquet à la houppe, ouvr. cité, p. 233.

47.Antoine de Léris, Dictionnaire portatif, historique et littéraires des théâtres [...], Paris, C.A. wombert, 1763, p. 663.

48.Rapportons l'ensemble de cette précieuse distributionæ: la Princesse était jouée par $\mathrm{M}^{\text {lle }}$ Silvia $;$; le Prince par Romagnesi $;$ la fée Bruyante par $\mathrm{M}^{\text {lle }}$ Belmont $\mathrm{x}$; la fée Agatine par $\mathrm{M}^{\text {lle }}$ Thomassin, Lisandre par Deshayes, Silvaine par $\mathrm{M}^{\text {lle }}$ Delalandex; Alcine par $\mathrm{M}^{\text {lle }}$ Thomassin la cadettex; ni les rôles d'Arlequin ni celui de l'Amour ne sont distribués. Mais on sait qu'Arlequin était alors interprété par Thomassin (Thomasso-Antonio Vicentini).

49.Antoine de Léris, ouvr. cité, p. 663.

50.Signalons l'étude de William H. Trapnell « The 'Philosophical' implications of Marivaux's Dispute » dans Studies on Voltaire and the Eighteenth Century, Genève, Institut et Musée Voltaire, Les Délices, 1970, t. 73, p. 193-219.

51. Voir notre Charles-François Pannard et l'esthétique du " petit ", Oxford, Fondation Voltaire, SVEC $2000: 01$, p. 246 et suiv.

52.Alzire connut également un succès éclatant, confirmé par les trois parodies que cette tragédie inspira coup sur coupx: à la Foire Saint-Germain, sous le titre Alzirette (opéracomique de... Pannard, Ponteau, Parmentier, créé le 18 février 1736) et aux Marionnettes de Bienfait sous le titre La Fille obéissante (par un anonyme) ; à la Comédie-Italienne, sous le titre Les Sauvages (parodie de... Romagnesi et Riccoboni, créée le 5 mars 1736).

53.François-Marie Arouet de Voltaire, Alzire ou Les Américains, Paris, Jean-BaptisteClaude Bauche, 1736.

54.Série qui constituerait un beau matériau d'étude pour le programme « Approche sérielle de la littérature du XVIII ${ }^{e}$ siècle » sous la direction de Sylvain Menant au CELLF $17^{\mathrm{e}}-18^{\mathrm{e}}$. 


\section{RÉSUMÉS}

Inspirée du conte Riquet à la houppe de Charles Perrault, la comédie Les Fées de Jean-Antoine Romagnesi et Michel Procope-Couteaux a figuré au répertoire de la Comédie Italienne pendant près de vingt ans à partir de 1736, année de sa création. Elle a été plusieurs fois imprimée et jouée à la Cour. Comment s'explique cette fortune exceptionnelle au regard des autres féeries dramatiques contemporaines, dont le succès, si éclatant fût-il, demeurait généralement sans lendemain?

Une analyse dramaturgique détaillée mettra à jour des éléments de réponse. En soulignant la manière "mêlée » de nos dramaturges, qui vont au-delà du merveilleux conventionnel pour inscrire leur pièce dans le sillage des comédies amoureuses de Marivaux, en particulier Arlequin poli par l'amour et La Seconde surprise de l'amour; en démontrant l'action souterraine, ample et puissante de la parole, qui se substitue tout au long de l'intrigue aux effets spectaculaires (ici relégués au second plan), et qui fait du langage même la force motrice de la pièce; en reliant enfin cette fable, où la primauté de l'expérience est consacrée et la foi inconditionnelle dans l'entendement humain attestée, aux réflexions philosophiques qui, à la même époque, contribuent à la propagation de l'empirisme de Locke et annoncent l'optimisme triomphant des Lumières.

\section{Les Fées of Romagnesi and Procope-Couteaux (1736) : between Perrault and Marivaux}

On the basis of Charles Perrault's tale, Riquet à la houppe (Ricky with the Tuft), Jean-Antoine Romagnesi and Michel Procope-Couteaux wrote a comedy, entitled Les Fées, which premiered in 1736 at the Comédie Italienne and remained for close to twenty years in the repertory of that theatre for close to twenty years. A few editions were printed and the play was performed several times at court. Why was it blessed with such an exceptional fate compared to the other contemporary fairy-tale-like theatrical productions, whose success, however bright it might have been, was never more than short-lived?

Our detailed dramaturgical analysis seeks to provide some elements of response through three different angles: emphasising the "mixed" approach of both dramatists, who strove much beyond the conventional supernatural element to set their play in the wake of Marivaux's love comedies, such as Arlequin poli par l'amour and La Seconde surprise de l'amour; illustrating the full scope and power of the spoken word, which supersedes spectacular effects (relegated here to a secondary position) throughout the plot, and which boosts language itself as the driving force of the play; and lastly, correlating that tale, in which the primacy of experience is consecrated and the unconditional faith in the human mind is validated, with philosophical reflections that contributed during that period to the dissemination of Locke's empiricism and led the way to the triumphant optimism of the Enlightenment. (Traduction : Gilles Plante.) 